\title{
Tooth loss in relation to serum cotinine levels - A cross-sectional study from the Belville South area in South Africa
}

SADJ May 2021, Vol. 76 No. 4 p207 - p215

F Kimmie-Dhansay ${ }^{1}$, CC Pontes ${ }^{2}$, U Chikte ${ }^{3}$, RT Erasmus ${ }^{4}$, AP Kengne ${ }^{5}$, TE Matsha ${ }^{6}$

\section{ABSTRACT}

\section{Introduction}

Tooth loss constitutes a major public health challenge, sharing common risk factors with non-communicable diseases.

\section{Aims and objectives}

To report the relationship between tooth loss and serum cotinine levels in a population sample of mixed ethnic heritage from the Belville South area in South Africa.

\section{Design}

Cross-sectional epidemiological study.

\section{Author affiliations:}

1. Faheema Kimmie-Dhansay: BSc BChD PDD PDD MSc MSc, a). Division of Research and Higher Degrees, University of the Western Cape, Cape Town, South Africa.

b). Division of Epidemiology and Biostatistics, Department of Global Health, Stellenbosch University, Cape Town, South Africa. ORCID Number: 0000-0003-2919-6193

2. Carla C Pontes: $D D S, M S c, P h D$, Division of Health Systems and Public Health, Department of Global Health, Stellenbosch University, Cape Town, South Africa.

ORCID Number: 0000-0002-9337-3783

3. Usuf Chikte: $B C h D, M S c$ (DPH), MDent (Community Dentistry) $P h D$, Department of Global Health, Stellenbosch University, Cape Town, South Africa.

ORCID Number: 0000-0002-7985-2278

4. Rajiv T Erasmus: MBBS, FMC.Path, FCPath (SA), DABCC (Am Board Certified), DHSM (Natal), Division of Clinical Pathology, Stellenbosch University, Cape Town, South Africa. ORCID Number: 0000-0001-6831-4215

5. Andre P Kengne: $M D, P h D$, Non-Communicable Diseases Research Unit, South African Medical Research Council, Cape Town, South Africa.

ORCID Number: 0000-0002-7213-9760

6. Tandi E Matsha: PhD, Cardio-metabolic Health Research Unit, Department of Biomedical Sciences, Faculty of Health and Wellness Science, Cape Peninsula University of Technology, Bellville, Cape Town, South Africa.

ORCID Number: 0000-0001-5251-030X

Corresponding author: Usuf Chikte

Department of Global Health, Stellenbosch University, Cape Town, South Africa.

Email: umec@sun.ac.za

Author contributions:

1. Faheema Kimmie-Dhansa: conceptualization, methodology, formal analysis - $16.6 \%$

2. Carla C Pontes: methodology, writing - original draft, review and editing - $16.6 \%$

3. Usuf Chikte: conceptualization, methodology, resources - 16.6\%

4. Rajiv T Erasmus: conceptualization, data curation, funding acquisition - $16.6 \%$

5. Andre $\mathbf{P}$ Kengne: project administration, validation, resources $-16.6 \%$

6. Tandi E Matsha: investigation, funding acquisition, data curation, resources - $16.6 \%$

\section{Methods}

Subjects were invited from 2014 to 2016 according to a consecutive sampling technique and all those who met the inclusion criteria were included.

\section{Results}

In all, 1876 individuals were included, being 1416 females (75.5\%), with a combined average age of $49.5 \pm 15.3$ years. In total $46.7 \%$ of the sample was edentulous, with females presenting a higher proportion than males $(50.7 \%$ vs. $34.1 \%, p<0.001)$.

The relative risk $(\mathrm{RR})$ of being edentulous was higher for females $(R R=1.8,95 \% \mathrm{Cl}=1.35-2.41, p<0.001)$ and for participants with cotinine levels $15-299 \mathrm{ng} / \mathrm{ml}(R R=1.37$, $95 \% \mathrm{Cl}=1.02=1.83, \mathrm{p}=0.04)$ and $\geq 300 \mathrm{ng} / \mathrm{ml}(\mathrm{RR}=1.51$, $95 \% \mathrm{Cl}=1.09-2.08, \mathrm{p}=0.01$ ). Maxillary incisors and mandibular molars were the most prevalent missing teeth.

\section{Conclusions}

The burden of tooth loss is high in the studied population sample, as well their unmet needs for dental care. Female gender, tobacco exposure, and aging were associated with partial and total edentulism.

\section{Keywords}

Tooth loss, edentulism, edentulousness, epidemiology oral conditions, serum cotinine.

\section{INTRODUCTION}

Optimal oral health is an essential part of systemic health. Worldwide, oral diseases constitute a burden for 3.5 billion people, being the most common non-communicable diseases (NCDs). ${ }^{1}$ Following dental caries and periodontal disease, tooth loss is the third most prevalent oral condition in the world. ${ }^{2}$ Loss of natural teeth can lead to physiological and psychosocial limitations, impairment, disability, and handicap, particularly in the absence of prosthetic replacement, having a strong impact on oral health-related quality of life $(\mathrm{OHRQOL}){ }^{3}$

A dentition with less than twenty teeth provides limited oral function, affecting chewing, speech, alveolar bone, and facial structures. ${ }^{2}$ Besides the local effects, complete edentulism has also been associated with nutritional deficiencies, ${ }^{4}$ gastrointestinal changes, ${ }^{5}$ kidney disease, ${ }^{6}$ cardiovascular disease, ${ }^{7}$ and increased mortality rates. ${ }^{8}$ 
Tooth loss remains a major public health challenge, constituting a debilitating and irreversible consequence of untreated caries and periodontitis in most cases. ${ }^{9}$

Oral conditions, including tooth loss, share important behavioural and social risk factors with other NCDs, such as unhealthy diet, consumption of tobacco products, and alcohol. ${ }^{10}$ Socioeconomic inequalities also affect oral diseases and tooth loss, with disadvantaged communities being particularly affected despite global improvements. ${ }^{10}$

In 2010, 158 million people (2.3\% of the global population) were edentulous. Prevalence of severe tooth loss reduced between 1990 and 2020 from $4.4 \%$ to 2.4\%. Global incidence also decreased from 374 cases per 100000 person-years in 1990 to 205 cases per 100000 person-years in $2010 .^{2}$

Over the last decades, there has been a decrease in the prevalence of edentulism in developed countries, however, there is limited data on tooth loss in developing countries, where health inequality remains high. ${ }^{11}$ In South Africa, the last national survey from 1988/1989 reported a prevalence of $51.6 \%$ of edentulism for adults of mixed ancestry aged 35-44 years, from which 3.5\% did not have dentures. ${ }^{12}$ To our knowledge, no studies have evaluated tooth loss in relation to serum cotinine levels and few epidemiological studies have reported tooth loss in the country, mostly through self-reported data. ${ }^{11,13}$

Cotinine constitutes the main metabolite of nicotine, a highly addictive chemical found in tobacco products. ${ }^{14}$ Given its long half-life, serum cotinine levels have been successfully validated and used in scientific studies as a biomarker for tobacco exposure. ${ }^{15}$ The present study included a convenient sample from the Belville South, a disadvantaged community located in the Western Cape Province in South Africa, characterized by a mixed ethnic background. Epidemiological data on tooth loss is essential to define treatment needs for public health policies. ${ }^{2}$ This study aimed to present epidemiological data on the relationship between tooth loss and serum cotinine levels in relation to age and gender for the Belville South area.

\section{MATERIALS AND METHODS}

\section{Sample}

This study was part of the Cape Town Belville South Vascular and Metabolic Health (VMH) study, which evaluated non-communicable diseases in the Belville South Community, Western Cape, South Africa, as described previously in detail. ${ }^{16}$

The Ethics Committee of the Faculty of Health and Wellness Sciences of the Cape Peninsula University of Technology provided ethical permission for the study (N14/ 01/003a) and it was conducted in agreement with the Declaration of Helsinki.

Subjects were invited from 2014 to 2016 according to a consecutive sampling technique and all those who met the inclusion criteria were included after signing the informed consent to participate in the study.
The inclusion criteria were: subjects 18 years or older with a mixed ethnic background (defined according to the South African Population Registration Act No. 30 of 1950, repealed in 1991) living in the Belville South area. The exclusion criteria included subjects requiring prophylactic use of antibiotics, with intellectual disabilities, with cancer, undergoing renal dialysis, pregnant, or those who were too ill or unable to give written consent. This study is STROBE compliant.

\section{Clinical examination}

One trained dental examiner used a portable dental chair with a portable overhead LED light to record clinical data. Standard infection control measures from the Centre for Disease Control were followed. Present and missing teeth were recorded for each patient and the use of dentures was recorded through a questionnaire. Third molars were not included in the study as their inclusion is optional. A reliability assessment was performed between the examiner and a calibrator through the Cohen's kappa statistic, with a $\kappa$ (kappa) score of 0.79 for decayed, missing, filled teeth, as reported previously. ${ }^{17}$

\section{Extent of tooth loss and posterior occluding pairs}

The extent of tooth loss was calculated as described previously: ${ }^{2}$ edentulous (no teeth present), severe tooth loss (1-9 teeth present), moderate tooth loss (10-19 teeth present), and functional dentition ( $\geq 20$ teeth present). The number of posterior occluding pairs was also recorded (presence of the tooth pairs 14/44, 15/45, $16 / 46,17 / 47,24 / 34,25 / 35,26 / 36$, and 27/47). Shortened dental arch (SDA) was classified as the number of teeth present from 15-25 and 35-45.

\section{Serum cotinine levels}

A fasting blood sample was collected for each participant and kept cold until being transported to an accredited laboratory. The samples were processed and evaluated for serum cotinine levels using a chemiluminescent assay (Immulite 1000, Siemens), as described previously. ${ }^{18}$ Cotinine constitutes the principal metabolite of nicotine, and it has been used as a biomarker for tobacco exposure due to its long half-life. ${ }^{15}$ The cut-off value of $15 \mathrm{ng} / \mathrm{ml}$ was used in the current study to differentiate smokers from non-smokers, according to results from previously published literature. ${ }^{14,19,20}$

\section{Statistical analysis}

Normality assumption of missing teeth variable was assessed through the Shapiro-Wilk test, which indicated that the data was skewed $(p<0.001)$. For categorical data, chi-square tests were employed, whereas for continuous data, Mann-Whitney and Kruskal Wallis tests were employed.

Variables were presented as percentage proportion or mean and standard deviation (SD) in relation to age group, gender, and serum cotinine levels in order to comply with the standard presentation of data from $\mathrm{WHO}$ and to allow for comparison to other studies. 
A multinomial logistic regression, which is the regression analysis used when the dependent variable is nominal with 2 or more levels, was used to explain the relationship between tooth loss category (dependent variable: edentulous, severe and moderate tooth loss and functional dentition), age, gender and serum cotinine category (independent variables).

Multinomial logistic regression was favoured over an ordered logistic regression since the proportional odds assumption was not met. ${ }^{21}$ Functional dentition was used as the reference category. Data were analysed using the Statistical Package STATA 15 (Stata Corp LP, College Station, TX, USA, 2017). The significance level was defined at $5 \%$.

\section{RESULTS}

The study consisted of 1888 individuals, from which 12 presented missing data. In total, 1876 individuals were included in the analysis, from which 1416 were females $(75.5 \%)$ and 460 (24.5\%) were males. The average age of the participants was $49.5 \pm 15.3$ years (range 1891). Data was evaluated according to the following age group categories: $>24$ years $(n=117,6.2 \%), 25-34$ years $(n=274,14.6 \%), 35-44$ years $(n=271,14.5 \%), 45-54$ $(n=407,21.7 \%), 55-64(n=491,26.2 \%)$ and $\geq 65$ years $(n=316,16.8 \%)$.

\section{Extent of tooth loss}

In total, $46.6 \%(n=875)$ of the sample was edentulous, $5.5 \%(n=104)$ had severe tooth loss (1-9 teeth present), $19.0 \%(n=357)$ had moderate tooth loss (10-19 teeth present) and $28.8 \%(n=540)$ had a functional dentition. Only $4 \%(n=77)$ of the sample had all teeth present and $41.7 \%$ presented a shortened dental arch (SDA).

Edentulousness was associated with gender, with $50.7 \%$ of females being edentulous, as compared to $34.1 \%$ of males $(p<0.001$, Figure 1$)$. It was also as- sociated with age group, with the highest proportion for the group aged 65 and above $(84.8 \%, n=268)$ and for the 55-64 years group $(74.1 \%, n=364)$.

With regards to edentulous participants, $12.9 \%(n=113)$ had no dentures, $87.0 \%(n=761)$ had upper and lower dentures and $0.1 \%(n=1)$ had top dentures. There was no association between use of dentures and gender (Table 1, p>0.05).

\begin{tabular}{|l|l|l|l|}
\hline \multicolumn{3}{|c|}{$\begin{array}{l}\text { Table 1. Use of dentures among edentulous participants } \\
\text { according to gender presented as \% proportion and count. }\end{array}$} \\
\hline & $\begin{array}{c}\text { No dentures } \\
\%(\mathbf{n})\end{array}$ & $\begin{array}{c}\text { Upper and lower } \\
\text { dentures } \\
\%(\mathbf{n})\end{array}$ & $\begin{array}{c}\text { Top dentures } \\
\%(\mathbf{n})\end{array}$ \\
\hline Males & $\mathbf{1 4 . 0 \% ( 2 2 )}$ & $85.4 \%(134)$ & $0.6 \%(1)$ \\
\hline Females & $12.7 \%(91)$ & $87.3 \%(627)$ & 0 \\
\hline Total & $12.9 \%(113)$ & $87.0 \%(761)$ & $0.1 \%(1)$ \\
\hline
\end{tabular}

Males had higher proportion of moderate tooth loss (22.6\% $n=104)$ as compared to females $(17.9 \%, n=253$, $\mathrm{p}=0.02)$. Moderate tooth loss was also associated with age group, with highest proportion observed in the group $35-44$ years $(35.1 \%, n=95, p<0.001)$.

In terms of severe tooth loss, the proportion was higher for males as compared to females $(7.6 \%$ vs. $4.9 \%$, $p=0.03)$. Severe tooth loss was associated with age group, with the group 45-54 years presenting the highest proportion $(9.6 \%, n=39, p<0.001)$.

There were more males with a functional dentition than females $(35.7 \%$ vs. $26.6 p<0.001)$. For the youngest group ( $<24$ years), $85.5 \%$ had a functional dentition and it decreased with aging, dropping to only $2.8 \%$ for the oldest group $(n=9, p<0.001)$.

The average number of teeth lost per person was $9.1 \pm$ 6.6 when edentulous participants were excluded (Table 2). There were no statistically significant differences between males $(9.2 \pm 6.9)$ and females $(9.1 \pm 6.4)$. The average number of teeth lost increased with aging, with the

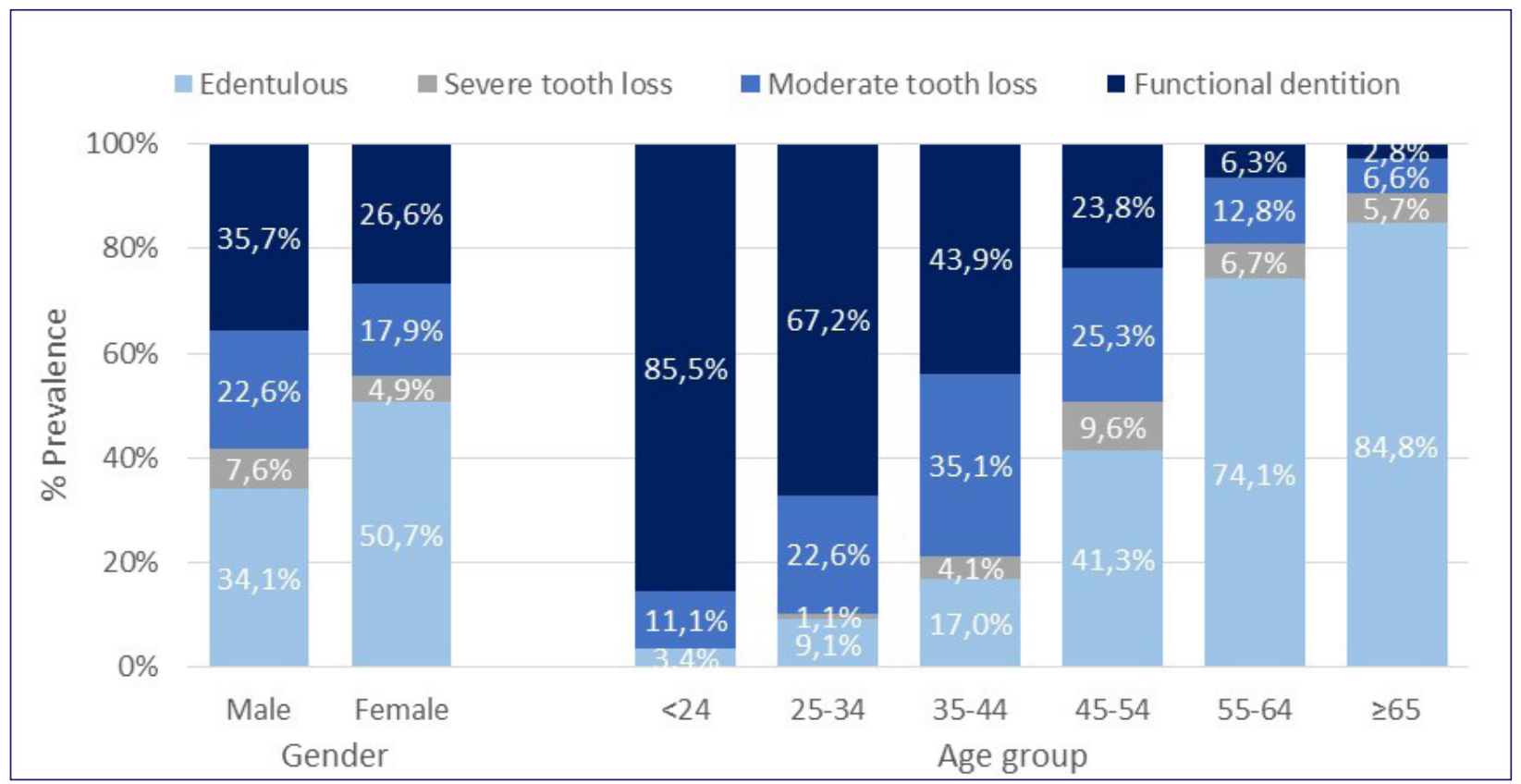

Figure 1. Extent of tooth loss according to gender and age group. 
youngest group ( $<24$ years) presenting $3.9 \pm 3.6$ teeth lost and the oldest group presenting $15.4 \pm 6.4$ teeth lost $(p<0.001$. Table 2$)$. The average number of missing teeth was nearly double for the maxilla, as compared to the mandible $(6.1 \pm 4.7$ vs. $3.1 \pm 3.0$, respectively. $p<0.001)$.

\begin{tabular}{|c|c|c|c|c|c|}
\hline & & \multicolumn{2}{|c|}{ Missing teeth } & \multicolumn{2}{|c|}{ Posterior occluding pairs } \\
\hline & & Mean & SD & Mean & SD \\
\hline \multicolumn{2}{|l|}{ Total } & 9.1 & 6.6 & 4.2 & 2.40 \\
\hline \multirow{2}{*}{ Gender } & Male $(n=303)$ & 9.2 & 6.9 & 4.1 & 2.60 \\
\hline & Female $(n=698)$ & 9.1 & 6.4 & 4.2 & 2.40 \\
\hline \multirow{6}{*}{$\begin{array}{l}\text { Age } \\
\text { group* }\end{array}$} & $<24(n=113)$ & 3.9 & 3.6 & 6.2 & 2.00 \\
\hline & $25-34(n=249)$ & 6.0 & 4.8 & 5 & 2.50 \\
\hline & $35-44(n=225)$ & 8.8 & 5.4 & 3.5 & 2.50 \\
\hline & $45-54(n=239)$ & 11.2 & 6.7 & 1.9 & 2.30 \\
\hline & $55-64(n=127)$ & 13.9 & 6.6 & 0.7 & 1.50 \\
\hline & $\geq 65(n=48)$ & 15.4 & 6.4 & 0.3 & 0.90 \\
\hline
\end{tabular}

Patterns of tooth loss

The four maxillary incisors were the most frequently missing teeth in this sample (55.8\%-54.6\% Figure 2), together with mandibular first molars (53.3-54.0\%). Lower incisors and canines were the least missing teeth (5.5-8.6\%).

There was no statistically significant difference between males and females regarding the average number of missing teeth according to tooth types (Figure 3 ).
Average number of missing teeth increased with increasing age group for all tooth types (Figure 4). In the youngest group ( $<24$ years), incisors had the highest average for missing teeth $(1.7 \pm 2.0)$. For the oldest group $(\geq 65$ years), molars were the most commonly missing teeth $(5.6 \pm 2.1)$.

\section{Posterior occluding pairs}

On average, dentate individuals had $3.3 \pm 2.9$ pairs of posterior occluding teeth and it decreased from $6.0 \pm 2.2$ for the youngest group to $0.7 \pm 1.3$ for the oldest group (Table 2). On average, males had $3.4 \pm 2.9$ and females had $3.2 \pm 2.9$ posterior occluding pairs.

When edentulous participants were excluded, 30.3\% $(n=303)$ of the sample had no posterior occluding pairs, $31.3 \%(n=313)$ had $1-4$ pairs and 38.3\% $(n=384)$ had 5-8 pairs (Figure 5). The number of occluding pairs was not associated with gender, but it was associated with age group $(p<0.001$, Figure 6$)$.

From the youngest $(<24$ years) to the oldest age group ( $\geq 65$ years), there was a drastic decrease in the proportion of 5-8 posterior occluding pairs, from $80.3 \%$ to $3.6 \%$. The number of participants without posterior occluding pairs increased from $4.3 \%$ in the youngest group to $72.8 \%$ in the oldest group.

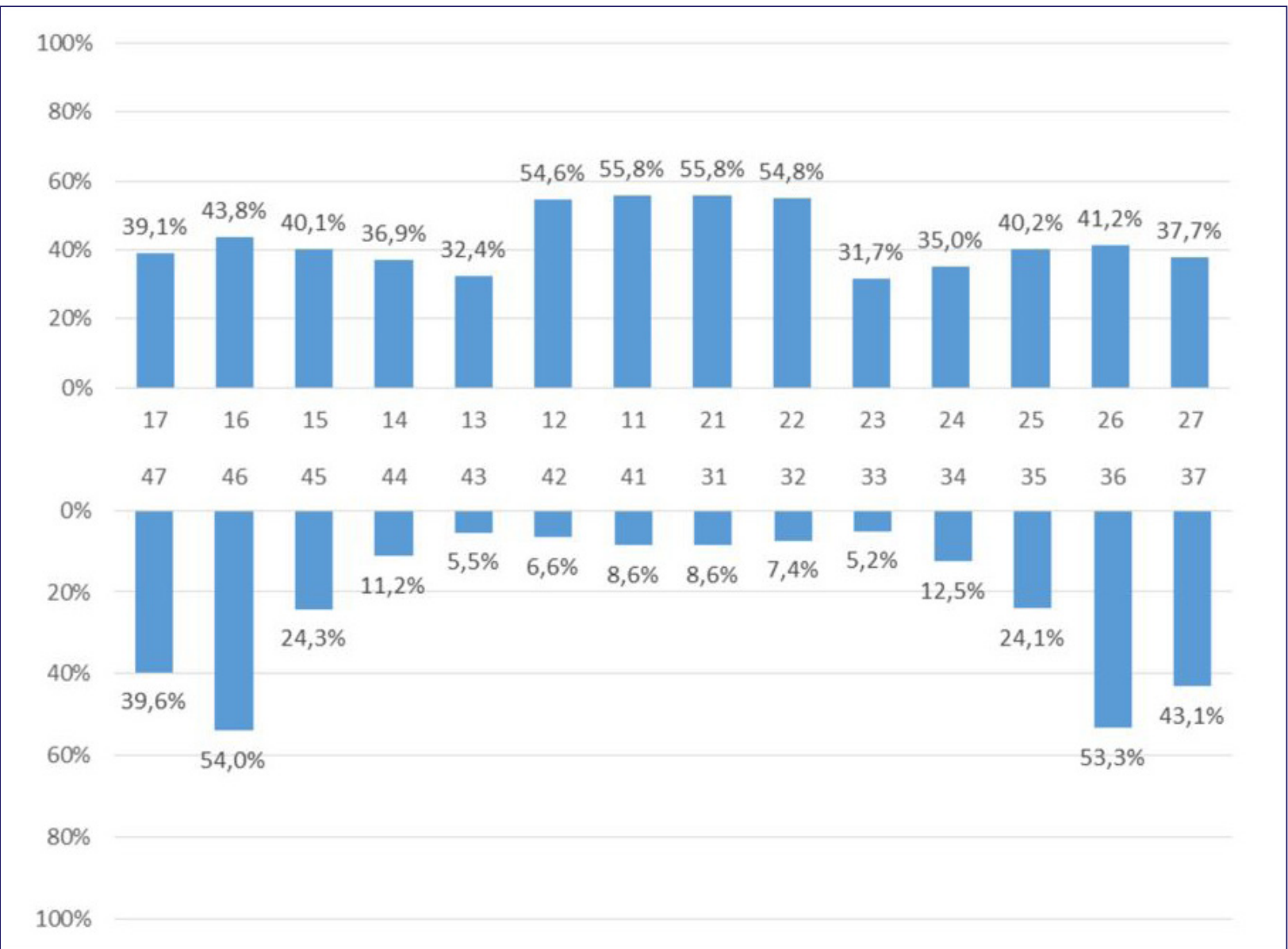

Figure 2. Prevalence of tooth loss according to tooth number in the maxilla and in the mandible. 


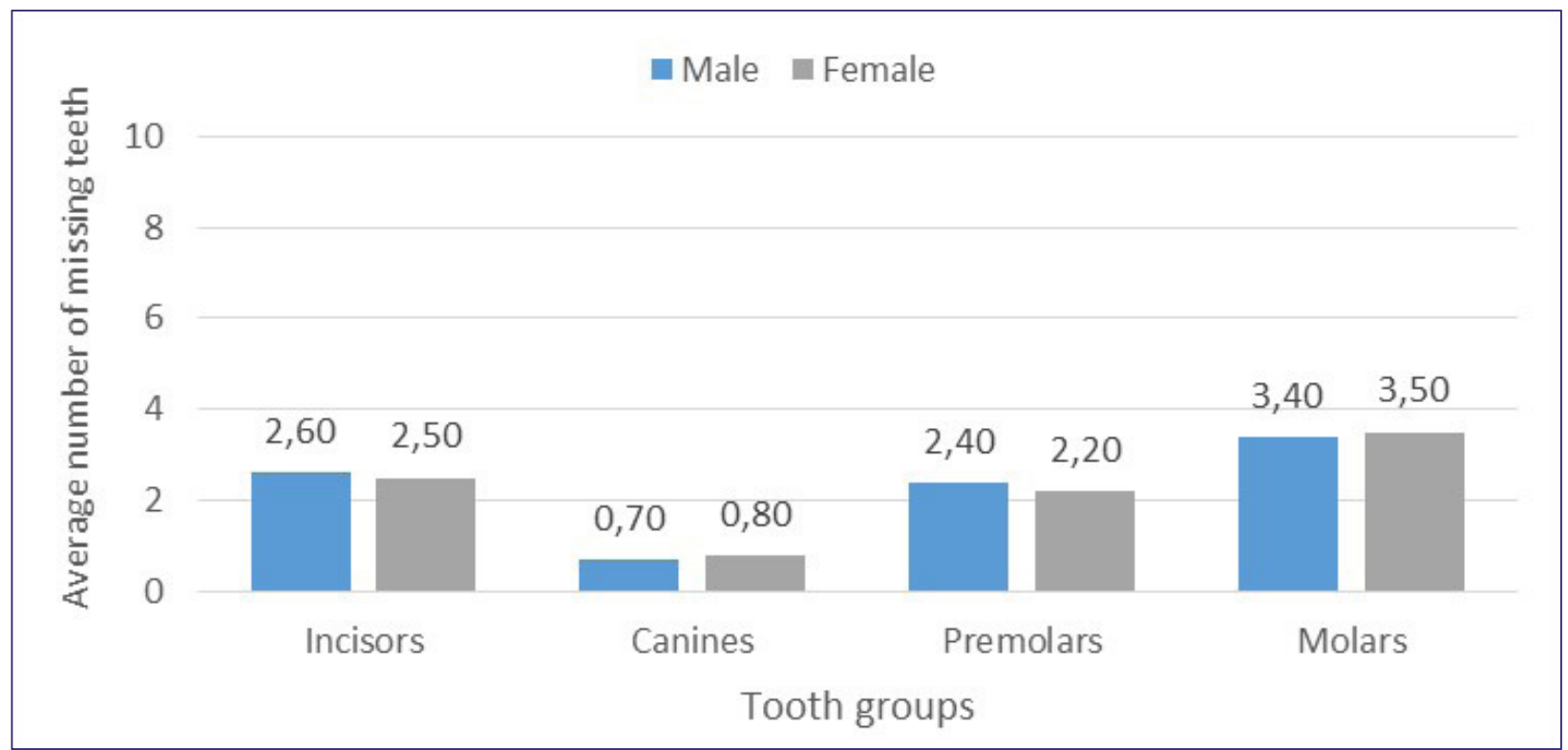

Figure 3. Average number of missing teeth according to tooth group by gender.

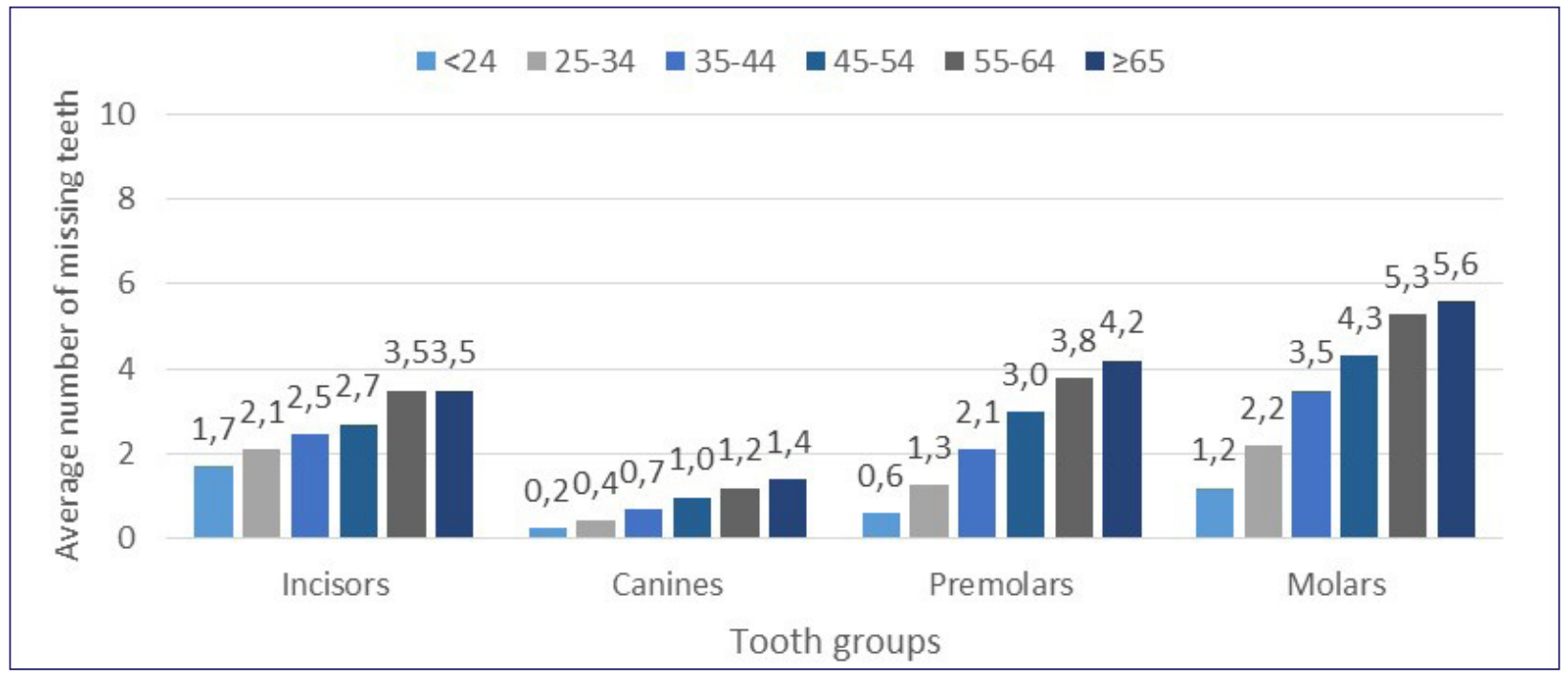

Figure 4. Average number of missing teeth according to tooth group by age group.

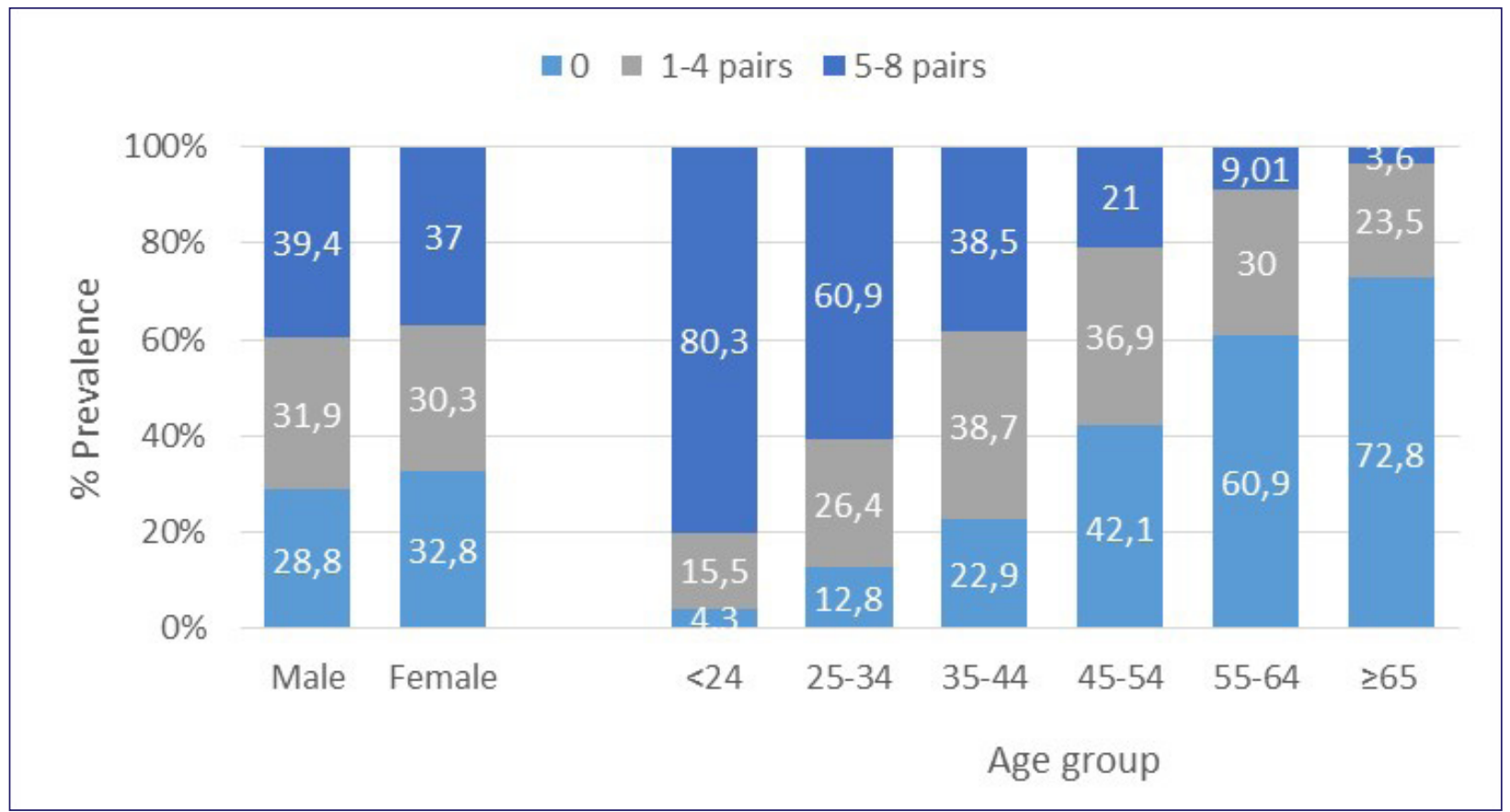

Figure 5. Prevalence of different categories of posterior occluding pairs according to gender and age group ( $p<0.001)$. 


\section{Serum cotinine}

Serum cotinine levels were measured in 1814 participants and categorized as $<15 \mathrm{ng} / \mathrm{ml}$ and $\geq 15 \mathrm{ng} / \mathrm{ml}$. From those, $51.0 \%$ had serum cotinine levels below $15 \mathrm{ng} / \mathrm{ml}(\mathrm{n}=-$ $924)$ and $49.0 \%(n=890)$ had levels $\geq 15 \mathrm{ng} / \mathrm{ml}$. Serum cotinine $\geq 15 \mathrm{ng} / \mathrm{ml}$ was observed in $59.7 \%$ of all men and $45.6 \%$ of all women.

Regarding age group, serum cotinine levels $\geq 15 \mathrm{ng} / \mathrm{ml}$ were observed in $61.3 \%$ of the youngest group, $65.8 \%$ of the $25-34,57.0 \%$ of the $35-44,52.0 \%$ of the $45-54$, $44.2 \%$ of the $55-64$ and $27.5 \%$ of the oldest group.

Cotinine categories were associated with tooth loss status, with most edentulous participants having cotinine levels below $15 \mathrm{ng} / \mathrm{ml}(56.6 \%, \mathrm{n}=479, \mathrm{p}<0.001)$ and most participants with severe tooth loss presenting cotinine levels $\geq 15 \mathrm{ng} / \mathrm{ml}(\mathrm{n}=54.8 \%, n=284, p=0.002$. Figure 6). There was no statistically significant association between posterior occluding pairs and cotinine categories.

\section{Logistic regression analysis}

The expected risk for being edentulous was higher for females. The relative risk of being edentulous, for females was $1.8(95 \% \mathrm{Cl}$ [1.35 to 2.41], $\mathrm{p}<0.001$, Table 3 ) when compared to males.

The expected risk of being edentulous was higher for participants with higher cotinine levels. The relative risk of being edentulous for serum cotinine levels $\geq 300 \mathrm{ng} / \mathrm{ml}$ was $1.51(95 \% \mathrm{Cl}$ [1.09 to 2.08], $\mathrm{p}=0.01$, Table 3) and 1.37 (95\% Cl [1.02 to 1.83], $p=0.04)$ for serum cotinine levels $15-299 \mathrm{ng} / \mathrm{ml}$, as compared to cotinine levels $<15$ $\mathrm{ng} / \mathrm{ml}$.

With a one-unit increase in age, there was an increase in the relative risk for participants being edentulous, having severe or moderate tooth loss.

\begin{tabular}{|c|c|c|c|c|}
\hline Tooth Loss Category & RR & P-value & \multicolumn{2}{|c|}{$95 \% \mathrm{Cl}$} \\
\hline Females & 1.81 & $<0.001$ & 1.35 & 2.42 \\
\hline \multicolumn{5}{|l|}{ Cotinine categories } \\
\hline $15-299 \mathrm{ng} / \mathrm{ml}$ & 1.37 & 0.04 & 1.02 & 1.83 \\
\hline$\geq 300 \mathrm{ng} / \mathrm{ml}$ & 1.51 & 0.01 & 1.10 & 2.08 \\
\hline Age & 1.12 & $<0.001$ & 1.11 & 1.13 \\
\hline Constant & 0.001 & $<0.001$ & 0.00 & 0.00 \\
\hline \multicolumn{5}{|l|}{ Severe tooth loss } \\
\hline Females & 0.68 & 0.20 & 0.39 & 1.20 \\
\hline \multicolumn{5}{|l|}{ Cotinine categories } \\
\hline $15-299 \mathrm{ng} / \mathrm{ml}$ & 1.83 & 0.05 & 0.99 & 3.43 \\
\hline$\geq 300 \mathrm{ng} / \mathrm{ml}$ & 1.11 & 0.79 & 0.50 & 2.47 \\
\hline Age & 1.07 & $<0.001$ & 1.05 & 1.09 \\
\hline Constant & 0.003 & $<0.001$ & 0.00 & 0.01 \\
\hline \multicolumn{5}{|l|}{ Moderate tooth loss } \\
\hline Females & 0.94 & 0.71 & 0.70 & 1.28 \\
\hline \multicolumn{5}{|l|}{ Cotinine categories } \\
\hline $15-299 \mathrm{ng} / \mathrm{ml}$ & 1.09 & 0.60 & 0.79 & 1.52 \\
\hline$\geq 300 \mathrm{ng} / \mathrm{ml}$ & 1.24 & 0.26 & 0.86 & 1.78 \\
\hline Age & 1.03 & $<0.001$ & 1.02 & 1.04 \\
\hline
\end{tabular}

\section{DISCUSSION}

To our knowledge, this is the first study reporting tooth loss in relation to serum cotinine levels in South Africa. Edentulism was highly prevalent and associated with female gender. It increased remarkably from the age of 45 and about $13 \%$ of edentulous participants had no dentures. Around $30 \%$ of the sample had a functional dentition, with a higher proportion in males. Maxillary incisors and mandibular molars were the most frequent missing teeth. One-third of all dentate participants had no posterior occluding pairs. Serum cotinine levels that suggest smoking were observed in approximately half of the sample and high cotinine levels were associated with edentulousness and severe tooth loss.

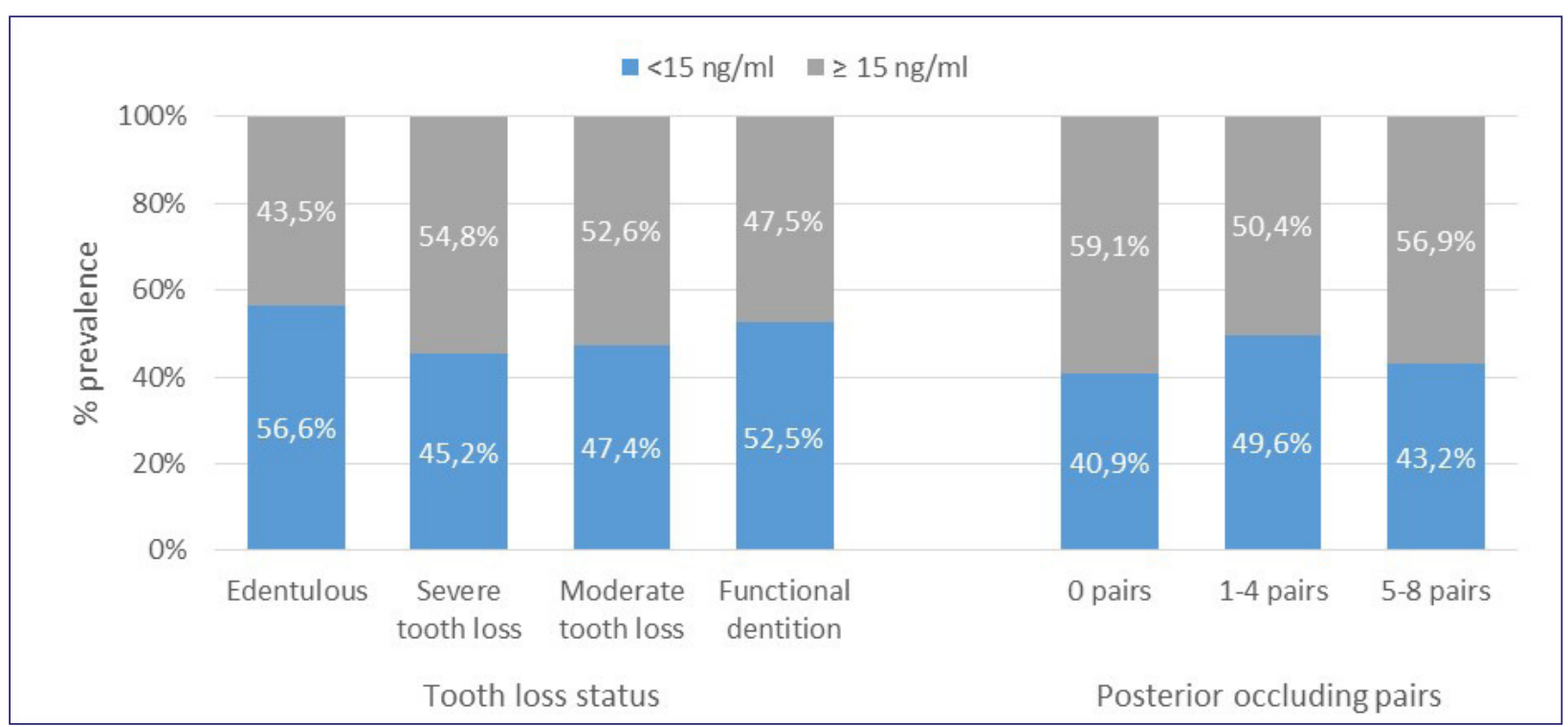

Figure 6. Serum cotinine categories according to tooth loss status ( $p<0.001$ for edentulous group, $p=0.002$ for severe tooth loss group) and number of posterior occluding pairs. 
According to the South African national survey from $1988 / 89$, the overall proportion of edentulism was $12.6 \%$, with a much higher proportion among adults aged 35-44 years with a mixed ethnic background (51.6\%). ${ }^{22}$ Considering the present finding that $46.7 \%$ of the sample was fully edentulous, total tooth loss remains a burden in the country, particularly for the population with a mixed ethnic background. In a study from Petersen \& Ogawa (2012), the authors reported the proportion of edentulism in subjects aged 65-74 in upper-middle-income countries to be $35 \%$, which is remarkably lower than the nearly $85 \%$ proportion reported for this age group in the current study. ${ }^{9}$

The worse situation of edentulism among females reported in this study (50.7\% of females vs. $34.1 \%$ for males) was comparable to the previously reported prevalence from the 1988/89 National Survey $(57.4 \%$ of females, vs. $41 \%$ of males) and is in accordance with studies from other middle-income countries, such as Brazil ${ }^{23}$ and Indonesia. ${ }^{24}$

It is likely that both biological, behavioural, and cultura factors influence the higher levels of tooth loss in females, including hormonal effects in the periodontium, higher caries, and extraction rate. ${ }^{25}$ In the present study, only $4 \%$ of the sample had all their teeth present, which is lower than the $6.6 \%$ reported in the last epidemiological South African national survey from 1988/89. ${ }^{26}$

Less than one-third of the sample had a functional dentition, defined as the minimum number of natural teeth present required for maintenance of masticatory function and quality of life. ${ }^{27}$ The striking decrease in the number of participants with a functional dentition after the age of 55 in this study suggests decreased quality of life and the chewing ability for the majority of participants in these age groups.

A study from Brazil reported the prevalence of functional dentition in participants aged $35-44$ years to be $54.6 \%$, which is somewhat comparable to the $43.9 \%$ reported in the present study for this age group. ${ }^{28}$ The authors also reported a higher prevalence of functional dentition in males, similarly to the present study.

Loss of posterior teeth can have a great impact on function. Having five or fewer posterior occluding pairs has a negative impact on oral impact on daily performance (OIDP) index. ${ }^{29}$ One third of the dentate sample included in the current study had no posterior occluding pairs and another one-third had 1-4 pairs, suggesting a reduced oral function for the majority of the sample.

The patterns of tooth loss in this study show that maxillary incisors were the most frequent missing teeth in younger participants. In older groups, first mandibular molars and maxillary incisors were the most frequent missing teeth. Extraction of healthy maxillary incisors has been reported previously as a common practice among the population included in the study, as part of gang activity and social factors. ${ }^{25}$

Nearly half of the sample in this study had serum cotinine levels compatible with smoking. Cotinine has been considered a reliable biomarker for tobacco exposure. ${ }^{30}$ While severe tooth loss was positively associated with higher serum cotinine, edentulousness was negatively associated with cotinine levels. From the observed trend of decreased cotinine levels with increased age, it can be speculated that as oral health deteriorates through tooth loss, tobacco consumption decreases.

Several predisposing factors can explain the high prevalence of edentulism observed. Lower-income has been linked to 2.5 higher odds of having tooth loss in comparison to higher income and low socioeconomic status has been associated with worse health behaviours and lack of access to dental care. ${ }^{31}$ Furthermore, high exposure to tobacco is also remarkable in the studied population, which is not only a risk factor for oral conditions, such as periodontal disease, tooth loss, and oral mucosal lesions, but is also a crucial risk factor for other NCDs, such as diabetes, cardiovascular disease, cancer, and respiratory diseases. ${ }^{32}$

The extraction of healthy maxillary incisors in communities of mixed ethnic ancestry in the Western Cape in South Africa has long been recognized as a common dental modification that also contributes to the high prevalence of missing teeth. ${ }^{33}$ In future studies, it would be interesting to evaluate a potential contribution of hypodontia to the burden of tooth loss in South Africa.

Replacement of missing teeth in individuals without a functional dentition is important to improve masticatory function, nutritional status, and quality of life in general. ${ }^{29}$ Social determinants of health also need to be addressed in order to improve the oral health status of disadvantaged communities. ${ }^{10}$ Implementation of strong tobacco prevention programs in middle-income countries such as South Africa needs to become a priority in terms of addressing common risk factors for tooth loss and other NCDs to improve quality of life. ${ }^{9}$ Promotion of oral health for this neglected population requires urgent attention in terms of policy-making.

\section{Limitations}

The high number of female participants can point to selection bias. Since clinical examination took place during working hours, employed males were likely hindered from participating. Because tooth loss is largely the end result of long-lasting dental and periodontal disease, estimates of associations between outcomes and exposures are difficult to establish through cross-sectional designs. Furthermore, the inclusion of potential confounders for tooth loss, such as alcohol consumption, oral hygiene habits, systemic conditions, and the exact cause for tooth extraction would have been beneficial.

The current study focused on a population group of mixed-ancestry, hence the results cannot be extrapolated to the entire South African population. Considering the heterogeneous prevalence of edentulism within countries and the lack of updated epidemiological data on tooth loss in South Africa, it is relevant to obtain data on other population groups in the country, particularly for underprivileged communities. In future studies, the relation between tooth loss and systemic diseases and 
conditions should be evaluated, considering the impact of edentulism on general health.

Lastly, recent epidemiological studies in the UK and US have used serum cotinine cut-off values as low as 3ng/ $\mathrm{ml}$ to distinguish between smokers and non-smokers. ${ }^{30}$ In the current study, the traditional cut-off value of $15 \mathrm{ng} /$ $\mathrm{ml}$ was used and the lowest value detected was $10 \mathrm{ng} / \mathrm{ml}$. Future studies should employ assays that have a lower detection range, as they can be valuable for detecting second-hand smoke.

\section{CONCLUSIONS}

Findings from the present study indicate that the burden of tooth loss is high in the studied population sample, as well their unmet needs for dental care. Female gender, tobacco exposure, and aging were associated with partial and total edentulism. The common approach to modifiable risk factors for NCDs, such as tobacco interventions, combined with access to preventive and restorative treatment has the potential to improve oral health status in this population sample.

\section{Acknowledgements}

We would like to thank all the participants of this study.

\section{Source of funding}

This project is the result of funding provided by the Medical Research Council of South Africa in terms of the MRC's Flagship's Awards Project SAMRC-RFA-UFSP $-01-2013 / \mathrm{VMH}$, received by $\mathrm{TM}$. The funders had no role in study design, data collection, and analysis, decision to publish, or preparation of the manuscript.

\section{Declaration}

The authors declare no conflict of interest.

\section{References}

1. Peres MA, D Macpherson LM, Weyant RJ, et al. Oral diseases: a global public health challenge. The Lancet. 2019; 394: 249-60. Doi: 10.1016/S0140-6736(19)31146-8.

2. Kassebaum NJ, Bernabé E, Dahiya M, et al. Global Burden of Severe Tooth Loss. J Dent Res. 2014; 93: 20S-28S. Doi: 10.1177/0022034514537828.

3. Ortíz-Barrios LB, Granados-García V, Cruz-Hervert P, et al. The impact of poor oral health on the oral health-related quality of life (OHRQOL) in older adults: the oral health status through a latent class analysis. BMC Oral Health. 2019; 19: 141. Doi: 10.1186/s12903-019-0840-3.

4. Carlsson GE, Jansson J-O, Österberg T, et al. Edentulism associated with obesity: a study of four national surveys of 16416 Swedes aged 55-84 years. Acta Odontol Scand. 2010; 68: 360-7. Doi: 10.3109/00016357.2010.514721.

5. Abnet CC, Qiao YL, Dawsey SM, et al. Tooth loss is associated with increased risk of total death and death from upper gastrointestinal cancer, heart disease, and stroke in a Chinese population-based cohort. Int J Epidemiol. 2005; 34: 467-74. Doi: 10.1093/ije/dyh375.

6. Fisher MA, Taylor GW, Shelton BJ, et al. Periodontal Disease and Other Nontraditional Risk Factors for CKD. Am J Kidney Dis. 2008; 51: 45-52. Doi: 10.1053/j.ajkd.2007.09.018.

7. Holmlund A, Holm G, Lind L. Number of teeth as a predictor of cardiovascular mortality in a cohort of 7,674 subjects followed for 12 years. J Periodontol. 2010; 81: 870-6. Doi: 10.1902/jop.2010.090680.

8. Holm-Pedersen P, Schultz-Larsen K, Christiansen N, et al. Tooth loss and subsequent disability and mortality in old age. J Am Geriatr Soc. 2008; 56: 429-35. Doi: 10.1111/j.15325415.2007.01602.x.

9. Petersen PE, Ogawa $H$. The global burden of periodontal disease: towards integration with chronic disease prevention and control. Periodontol 2000. 2012; 60: 15-39. Doi: 10.11 11/j.1600-0757.2011.00425.x.

10. Watt RG, Daly B, Allison $P$, et al. Ending the neglect of global oral health: time for radical action. Lancet. 2019: 261-72. Doi: 10.1016/S0140-6736(19)31133-X.

11. Kailembo A, Preet R, Stewart Williams J. Common risk factors and edentulism in adults, aged 50 years and over, in China, Ghana, India and South Africa: Results from the WHO Study on global AGEing and adult health (SAGE). BMC Oral Health. 2016; 17. Doi: 10.1186/s12903-016-0256-2.

12. van Wyk PJ, van Wyk C. Oral health in South Africa. Int Dent J. 2004; 54: 373-7. Doi: 10.1111/j.1875-595X.2004.tb00014.x.

13. Peltzer K, Hewlett S, Yawson AE, et al. Prevalence of loss of all teeth (Edentulism) and associated factors in older adults in China, Ghana, India, Mexico, Russia and South Africa. Int J Environ Res Public Health. 2014; 11: 11308-24. Doi: 10.3390/ ijerph111111308.

14. Benowitz NL, III PJ, Ahijevych K, et al. Biochemical verification of tobacco use and cessation. Nicotine Tob Res. 2002; 4:149-59. Doi: 10.1080/14622200210123581.

15. Al-Bayaty F, Baharuddin N, Abdulla M. The relationship between serum cotinine levels and periodontal status. Online Journal of Biological Sciences. 2010; 10(2): 54-9.

16. Matsha TE, Hassan MS, Kidd M, et al. The 30-year cardiovascular risk profile of South Africans with diagnosed diabetes, undiagnosed diabetes, pre-diabetes or normoglycaemia: the Bellville, South Africa pilot study. Cardiovasc J Afr. 2012; 23: 5-11. Doi: 10.5830/CVJA-2010-087.

17. Chikte U, Pontes CC, Karangwa I, et al. Dental caries in a South African adult population: findings from the Cape Town Vascular and Metabolic Health Study. Int Dent J. 2020; 70(3): 176-82.

18. Matsha TE, Soita DJ, Hassan MS, et al. Three-year's changes in glucose tolerance status in the Bellville South cohort: Rates and phenotypes associated with progression. Diabetes Res Clin Pract. 2013; 99: 223-30. Doi: 10.1016/j.diabres. 2012. 10.018. 
19. Bunaes DF, Mustafa M, Mohamed HG, et al. The effect of smoking on inflammatory and bone remodeling markers in gingival crevicular fluid and subgingival microbiota following periodontal therapy. J Periodontal Res. 2017; 52: 713-24. Doi: 10.1111/jre.12438.

20. Jarvis MJ, Tunstall-Pedoe $\mathrm{H}$, Feyerabend $\mathrm{C}$, et al. Comparison of tests used to distinguish smokers from nonsmokers. Am J Public Health. 1987; 77: 1435-8. Doi: 10.2105/AJPH.77.11. 1435.

21. Lee E. Ordinal Logistic Regression and its Assumptions Full Analysis. Available at: https://medium.com/evangelinelee/ ordinal-logistic-regression-on-world-happiness-report-22137 2709095.

22. van Wyk PJ, van Wyk C. Oral health in South Africa. Int Dent J. 2004; 54: 373-7.

23. Ribeiro CG, Cascaes AM, Silva AER, et al. Edentulism, severe tooth loss and lack of functional dentition in elders: A study in Southern Brazil. Braz Dent J. 2016; 27: 345-52. Doi: 10.1590/0103-6440201600670.

24. Pengpid S, Peltzer K. The prevalence of edentulism and their related factors in Indonesia, 2014/15. BMC Oral Health. 2018; 18. Doi: 10.1186/s12903-018-0582-7.

25. Russell SL, Gordon S, Lukacs JR, et al. Sex/gender differences in tooth loss and edentulism. Historical perspectives, biological factors, and sociologic reasons. Dent Clin North Am. 2013: 317-37. Doi: 10.1016/j.cden.2013.02.006.

26. Government of South Africa. The dental caries status of the coloured population in the major metropolitan areas of the republic of South Africa. National Oral Health Survey, South Africa 1988/89. Department of Health. 2004.
27. WHO: Oral health surveys: basic methods $-5^{\text {th }}$ edition. WHO. 2016.

28. Koltermann AP, Giordani JM do A, Pattussi MP. A associação entre aspectos individuais e contextuais na dentição funcional de adultos do Rio Grande do sul, Brasil: Um estudo multinível. Cad Saude Publica. 2011; 27: 173-82. Doi: 10.1590/S0102-311X2011000100018.

29. Ferreira RC, Kawachi I, Souza JGS, et al. Is reduced dentition with and without dental prosthesis associated with oral health-related quality of life? A cross-sectional study. Health Qual Life Outcomes. 2019; 17: 79. Doi: 10.1186/s12955019-1149-2.

30. Kim S. Overview of cotinine cutoff values for smoking status classification. Int J Environ Res Public Health. 2016; 13(12): 1236. Doi: 10.3390/ijerph13121236.

31. Seerig LM, Nascimento GG, Peres MA, et al. Tooth loss in adults and income: Systematic review and meta-analysis. J Dent. 2015; 1051-9. Doi: 10.1016/j.jdent.2015.07.004

32. Glantz S, Gonzalez M. Effective tobacco control is key to rapid progress in reduction of non-communicable diseases. Thw Lancet. 2012; 1269-71. Doi: 10.1016/S0140-6736(11) 60615-6.

33. Friedling LJ, Morris AG. Pulling teeth for fashion: dental modification in modern day Cape Town, South Africa. SADJ. 2007; 62: 106, 108-113.

\section{Do the CPD questionnaire on page 226}

The Continuous Professional Development (CPD) section provides for twenty general questions and five ethics questions. The section provides members with a valuable source of CPD points whilst also achieving the objective of CPD, to assure continuing education. The importance of continuing professional development should not be underestimated, it is a career-long obligation for practicing professionals.

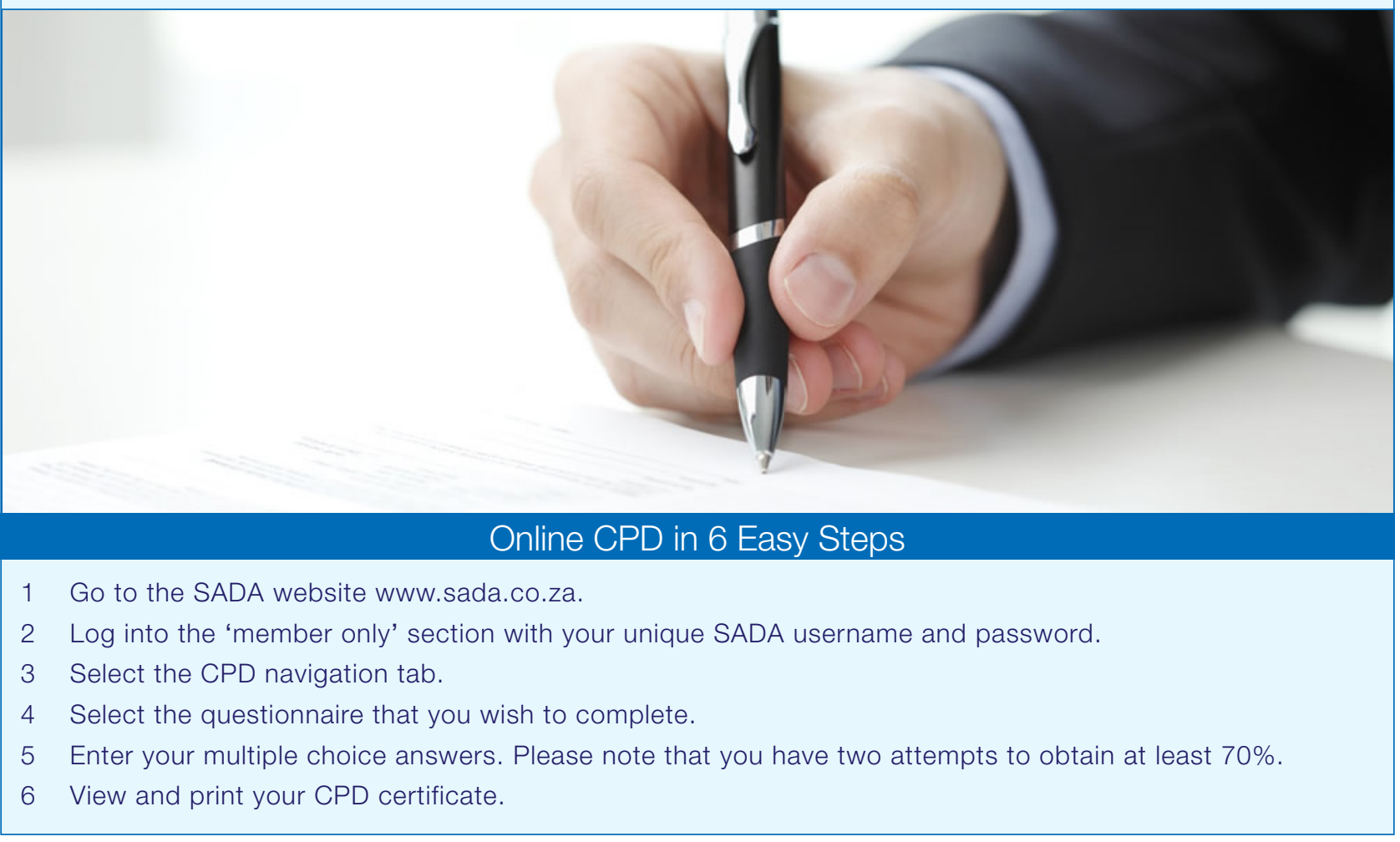

